\title{
The Cauchy problem and Hadamard's example
}

\author{
E. Mogileva* and O. Yaremko \\ Penza State University, 440026, Penza, Russia \\ ${ }^{*}$ Corresponding author E-mail: ElenaSergIvan@yandex.ru
}

\begin{abstract}
Integral representation for harmonic function in the ring. We prove the existence and uniqueness of solutions of the Cauchy problem for the Laplace equation in the circle. Integral representation for the solution of the Cauchy problem was found.
\end{abstract}

Keywords: Cauchy problem, examine Hadamard's.

\section{Introduction}

We now examine Hadamard's example of an ill-posed problem [1, 2]. Consider Cauchy problem for the Laplace's equation

$$
\begin{gathered}
\Delta u \equiv \frac{1}{r} \frac{\partial}{\partial r}\left(r \frac{\partial u}{\partial r}\right)+\frac{1}{r^{2}} \frac{\partial^{2} u}{\partial \varphi^{2}}=0 \\
\left.u\right|_{r=1}=0,\left.\frac{\partial u}{\partial n}\right|_{r=1}=e^{-\sqrt{n} \frac{\cos n \varphi}{n}}
\end{gathered}
$$

$n$ is an add integer. Then

$$
u(r, \varphi)=e^{-\sqrt{n}} \frac{r^{n}-r^{-n}}{2 n^{2}} \cos (n \varphi)
$$

is the unique solution Cauchy problem. But now consider large $n$. We have

$$
\left\|e^{-\sqrt{n}} \frac{\cos n \varphi}{n}\right\| \rightarrow 0
$$

for any Sobolev norm [3] because the beats all polynomials in $\mathrm{n}$ (from any finite order of derivatives). Hence we can choose $n$ so large that the boundary condition is as close to 0 as we please. But the solution $u(r, \varphi)$ grows large at any fixed line $r=$ constant as $n \rightarrow \infty$;

$$
\left\|u(r, \varphi)-u_{0}\right\| \rightarrow \infty
$$

hence $u_{0} \equiv 0$ solves

$$
\begin{aligned}
& \Delta u \equiv \frac{1}{r} \frac{\partial}{\partial r}\left(r \frac{\partial u}{\partial r}\right)+\frac{1}{r^{2}} \frac{\partial^{2} u}{\partial \varphi^{2}}=0 \\
& \left.u\right|_{r=1}=0,\left.\frac{\partial u}{\partial n}\right|_{r=1}=0
\end{aligned}
$$

uniquely. So this problem does not depend continuously on the data, and hence is not well-posed. Therefore it is not something we can safely ignore, well- posedness. 
In this paper the analytical solution of the Hadamard problem [4] in a ring is resulted. The received results can be applied for solving inverse boundary problems, type of the Hadamard problem. Let us arrive at the function $u(r, \varphi)$ such that $u$ is the solution of the Laplace's equation

$$
\Delta u \equiv \frac{1}{r} \frac{\partial}{\partial r}\left(r \frac{\partial u}{\partial r}\right)+\frac{1}{r^{2}} \frac{\partial^{2} u}{\partial \varphi^{2}}=0
$$

in the $\operatorname{ring} K: r<|z|<1 ; z=r e^{i \varphi}, u(r, \varphi$ is continuous in the closed ring $\bar{K}$. Moreover, for the function $u$ the conditions hold

$$
\left.u\right|_{r=1}=g(\varphi),\left.\quad \frac{\partial u}{\partial n}\right|_{r=1}=h(\varphi),
$$

$h(\varphi)$ is continuous, $2 \pi$ - periodic function. Let us show that the Hadamard problem solution is

$$
\begin{aligned}
& u(r, \varphi)=\frac{1}{2 \pi} \int_{0}^{\infty} e^{-\varepsilon} \int_{0}^{2 \pi} R e\left[\exp \left(\varepsilon z e^{-i t}\right)\right] g(t) d t+ \\
& +\frac{1}{2 \pi} \int_{0}^{\infty} e^{-\varepsilon} \int_{0}^{2 \pi} R e\left[\frac{1}{z} \exp \left(\varepsilon \frac{e^{i t}}{z}\right)\right] g(t) d t-\frac{1}{2 \pi} \int_{0}^{2 \pi} h(\psi) d \psi \cdot \ln r+ \\
& +\frac{1}{2 \pi} \int_{0}^{\infty} \frac{1}{\lambda}\left(e^{-\frac{\lambda}{r}}-e^{-\lambda r}\right) \int_{0}^{2 \pi} e^{\lambda \cos (\varphi-\psi)} \cos (\lambda \sin (\varphi-\psi)) h(\psi) d \psi d \lambda .
\end{aligned}
$$

\section{The main lemma}

Lemma 2.1 If the function $w=f(z)$ is analytic in the ring $r<|z|<1$, and continuous in the closed ring $r \leq|z| \leq 1$, then

$$
\begin{aligned}
& f(z)=\frac{1}{2 \pi i} \int_{0}^{\infty} e^{-\varepsilon} \int_{C_{r_{1}}} e^{\varepsilon \frac{z}{\varsigma}} f(\varsigma) \frac{d \varsigma}{\varsigma}+ \\
& +\frac{1}{2 \pi i} \int_{0}^{\infty} e^{-\varepsilon} \int_{C_{r_{2}}} \frac{1}{z} e^{\varepsilon \frac{\varsigma}{z}} f(\varsigma) d \varsigma, r<|z|<1 .
\end{aligned}
$$

Proof: Consider the sequence of function

$$
\begin{aligned}
& f_{N}(z)=\frac{1}{2 \pi i} \int_{0}^{N} e^{-\varepsilon} \int_{C_{r_{1}}} e^{\varepsilon \frac{z}{\varsigma}} f(\varsigma) \frac{d \varsigma}{\varsigma}+ \\
& +\frac{1}{2 \pi i} \int_{0}^{N} e^{-\varepsilon} \int_{C_{r_{2}}} \frac{1}{z} e^{\varepsilon \frac{\varsigma}{z}} f(\varsigma) d \varsigma, r<|z|<1 .
\end{aligned}
$$

From the Cauchy formula [6] it follows that the contour of integration can be transferred to a circles $C_{1}, C_{r}$. We get

$$
\begin{aligned}
& f_{N}(z)=\frac{1}{2 \pi i} \int_{0}^{N} e^{-\varepsilon} \int_{C_{1}} e^{\varepsilon \frac{z}{\varsigma}} f(\varsigma) \frac{d \varsigma}{\varsigma}+ \\
& +\frac{1}{2 \pi i} \int_{0}^{N} e^{-\varepsilon} \int_{C_{2}} \frac{1}{z} e^{\varepsilon \frac{\varsigma}{z}} f(\varsigma) d \varsigma, r<|z|<1 .
\end{aligned}
$$


Interchanging the order of integration in each summand and integrating the inner integrals with respect to $\varepsilon$, we obtain

$$
-\frac{1}{2 \pi i} \int_{C_{r}}\left[\frac{1}{\varsigma-z}-\frac{e^{-N\left(1-\frac{\varsigma}{z}\right)}}{\varsigma-z}\right] f(\varsigma) d \varsigma, r<|z|<1 .
$$

We pass to the limit as $N \rightarrow \infty$ and get the following estimates:

$$
\begin{aligned}
& \left|\frac{1}{2 \pi i} \int_{C_{1}}\left[\frac{e^{-N\left(1-\frac{z}{\varsigma}\right)}}{\varsigma-z}\right] f(\varsigma) d \varsigma\right| \leq \frac{1}{2 \pi} \int_{C_{1}} \frac{e^{-N(1-|z|)}}{1-|z|}|f(\varsigma)||d \varsigma| \leq \frac{e^{-N(1-|z|)}}{1-|z|} M \\
& \left|\frac{1}{2 \pi i} \int_{C_{r}}\left[\frac{e^{-N\left(1-\frac{\varsigma}{z}\right)}}{\varsigma-z}\right] f(\varsigma) d \varsigma\right| \leq \frac{1}{2 \pi} \int_{C_{r}} \frac{e^{-N\left(1-\frac{r}{|z|}\right)}}{|z|-r}|f(\varsigma)||d \varsigma| \leq \frac{e^{-N\left(1-\frac{r}{|z|}\right)}}{|z|-r} M \\
& M=\max _{r \leq|z| \leq 1}|f(z)| .
\end{aligned}
$$

From the estimates it follows that the summands

$$
\frac{1}{2 \pi i} \int_{C_{1}}\left[\frac{e^{-N\left(1-\frac{z}{\varsigma}\right)}}{\varsigma-z}\right] f(\varsigma) d \varsigma, \frac{1}{2 \pi i} \int_{C_{r}} \frac{e^{-N\left(1-\frac{\varsigma}{z}\right)}}{\varsigma-z} f(\varsigma) d \varsigma, r<|z|<1,
$$

tend to 0 . This is uniform convergence in $z$ in any of the closed $\operatorname{ring} r_{1} \leq|z| \leq r_{2}$. Thus we have $\lim _{N \rightarrow \infty} f_{N}(z)=f(z)$.

\section{$3 \quad$ Main results}

Theorem 3.1 Let us arrive at the function $u(r, \varphi)$ such that $u$ is the solution of the Laplace's equation

$$
\Delta u \equiv \frac{1}{r} \frac{\partial}{\partial r}\left(r \frac{\partial u}{\partial r}\right)+\frac{1}{r^{2}} \frac{\partial^{2} u}{\partial \varphi^{2}}=0
$$

in the ring $K: r<|z|<1, u$ is continuous in the closed ring $\bar{K}$. Moreover, for the function $u$ the conditions hold

$$
\left.u\right|_{r=1}=g(\varphi),\left.\quad \frac{\partial u}{\partial n}\right|_{r=1}=0,
$$

$g(\varphi)$ is continuous, $2 \pi$-periodic function, the solution of the problem (7)-(8) is expressed in the form :

$$
\begin{aligned}
& u(r, \varphi)=\frac{1}{2 \pi} \int_{0}^{\infty} e^{-\varepsilon} \int_{0}^{2 \pi} R e\left[\exp \left(\varepsilon z e^{-i t}\right)\right] g(t) d t+ \\
& +\frac{1}{2 \pi} \int_{0}^{\infty} e^{-\varepsilon} \int_{0}^{2 \pi} \operatorname{Re}\left[\frac{1}{z} \exp \left(\varepsilon \frac{e^{i t}}{z}\right)\right] g(t) d t, r<|z|<1 \\
& z=r e^{i \varphi}
\end{aligned}
$$

Proof: By the Cauchy - Riemann equations [5], so that $-i v_{\varphi}^{\prime}=r u_{r}^{\prime}$. We get $v(1, \varphi)=0$. It follows that we get $\left.f\right|_{r=1}=g(\varphi)$ for the function $f=u+i v$.

Substituting $r_{1}=r_{2}=1$ in (4), we obtain

$$
\begin{aligned}
& f(z)=\frac{1}{2 \pi} \int_{0}^{\infty} e^{-\varepsilon} \int_{0}^{2 \pi} \exp \left(\varepsilon z e^{-i t}\right) g(t) d t+ \\
& +\frac{1}{2 \pi} \int_{0}^{\infty} e^{-\varepsilon} \int_{0}^{2 \pi} \frac{1}{z} \exp \left(\varepsilon \frac{e^{i t}}{z}\right) g(t) d t, r<|z|<1 .
\end{aligned}
$$

We take a real part of $f(z)$. Theorem is proved. 
Theorem 3.2 Let us arrive at the function $u(r, \varphi)$ such that $u$ is the solution of the Laplace's equation

$$
\Delta u \equiv \frac{1}{r} \frac{\partial}{\partial r}\left(r \frac{\partial u}{\partial r}\right)+\frac{1}{r^{2}} \frac{\partial^{2} u}{\partial \varphi^{2}}=0
$$

in the ring $K: r<|z|<1$, u is continuous in the closed ring $\bar{K}$. Moreover, for the function $u$ the conditions hold

$$
\left.u\right|_{r=1}=0,\left.\quad \frac{\partial u}{\partial n}\right|_{r=1}=h(\varphi),
$$

$g(\varphi)$ is continuous, $2 \pi$-periodic function, The solution of the problem (7)-(8) is expressed in the form:

$$
\begin{aligned}
& u(r, \varphi)=-\frac{1}{2 \pi} \int_{0}^{2 \pi} h(\psi) d \psi \cdot \ln r+ \\
& +\frac{1}{2 \pi} \int_{0}^{\infty} \frac{1}{\lambda}\left(e^{-\frac{\lambda}{r}}-e^{-\lambda r}\right) \int_{0}^{2 \pi} e^{\lambda \cos (\varphi-\psi)} \cos (\lambda \sin (\varphi-\psi)) h(\psi) d \psi d \lambda . \\
& z=r e^{i \varphi} .
\end{aligned}
$$

Proof: Consider the Cauchy auxiliary problem:

$$
\begin{aligned}
& \Delta \tilde{u} \equiv \frac{1}{r} \frac{\partial}{\partial r}\left(r \frac{\partial \tilde{u}}{\partial r}\right)+\frac{1}{r^{2}} \frac{\partial^{2} \tilde{u}}{\partial \varphi^{2}}=0 \\
& \left.\tilde{u}\right|_{r=1}=g(\varphi),\left.\frac{\partial \tilde{u}}{\partial n}\right|_{r=1}=0
\end{aligned}
$$

where

$$
g(\varphi)=\frac{1}{2 \pi} \int_{0}^{2 \pi} \psi h(\varphi+\psi) d \psi .
$$

The solution of the problem (7)-(8) is by theorem 2 :

$$
\begin{aligned}
& u(r, \varphi)=\frac{1}{2 \pi} \int_{0}^{\infty} e^{-\varepsilon} \int_{0}^{2 \pi} \operatorname{Im}\left[\exp \left(\varepsilon z e^{-i t}\right)\right] g(t) d t+ \\
& +\frac{1}{2 \pi} \int_{0}^{\infty} e^{-\varepsilon} \int_{0}^{2 \pi} \operatorname{Im}\left[\frac{1}{z} \exp \left(\varepsilon \frac{e^{i t}}{z}\right)\right] g(t) d t, r<|z|<1 .
\end{aligned}
$$

Integrating by parts, and using

$$
\begin{aligned}
& g^{\prime}(\varphi)=h(\varphi) \\
& \widetilde{u}(r, \varphi)=\frac{1}{2 \pi} \int_{0}^{2 \pi} \psi u(r, \varphi+\psi) d \psi,
\end{aligned}
$$

we get (3). 


\section{References}

[1] Lavrentev M.M., Romanov V.G. and Shishatskii S.P., "Ill-Posed Problems of Mathematical Physics and Analysis.", American Mathematical Society, Providence,, (1986).

[2] Lavrentev M.M., Reznitskay K.G., Yakhno V.G., "One-Dimensional Inverse Problems of Mathematical Physics.",American Mathematical Society Translations, Series 2, Vol. 130, (1986).

[3] Nikol'skii, S.M. ,Sobolev space, in Hazewinkel, Michiel, Encyclopedia of Mathematics, Springer, ISBN 978-1556080104, (2001).

[4] A.N. Tychonov, V. Y. Arsenin , "Solution of Ill-posed Problems.",Washington: Winston and Sons. ISBN 0-470-99124-0 (1977).

[5] Lavrentev M.A., Shabat B.V., "Metods of Theory of Functions of a Complex Variable (in Russian)",Nauka, Moscow, (1973).

[6] Ahlfors, Lars (1979), Complex analysis (3rd ed.), McGraw Hill, ISBN 978-0070006577. 